\title{
Lien social et Politiques
}

Lien social

et Politicues

\section{Le féminisme et la question des " mères travailleuses ". Retour sur le tournant des années 1970. \\ Feminism and Working Mothers: Reassessing the Turning Point of the 1970s \\ El feminismo y el problema de « las madres trabajadoras ». Retorno a los años 70}

\section{Louise Toupin}

Numéro 36, automne 1996

Politiques familiales et vies de femmes

URI : https://id.erudit.org/iderudit/005241ar

DOI : https://doi.org/10.7202/005241ar

Aller au sommaire du numéro

Éditeur(s)

Lien social et Politiques

ISSN

1204-3206 (imprimé)

1703-9665 (numérique)

Découvrir la revue

Citer cet article

Toupin, L. (1996). Le féminisme et la question des " mères travailleuses ». Retour sur le tournant des années 1970. Lien social et Politiques, (36), 69-73.

https://doi.org/10.7202/005241ar

\section{Résumé de l'article}

Dans cet article, l'auteure entend soumettre à l'examen l'idée selon laquelle le féminisme aurait légitimé le modèle de la mère-travailleuse-salariée. Après un bref retour sur le contexte idéologique de la résurgence du féminisme sur la scène publique en Occident à la fin des années 1960, l'auteure estime qu'une partie seulement du mouvement a légitimé un tel modèle, alors qu'une autre partie, plus radicale, aurait plutôt — s'il faut parler en termes de légitimation - légitimé la révolte et la lutte des femmes contre leur double journée de travail et dénoncé le mythe de la libération par le travail salarié. 


\section{Le féminisme et la question des «mères travailleuses». Retour sur le tournant des années 1970}

\section{Louise Toupin}

Que ce soit en Amérique du nord ou dans plusieurs pays d'Europe occidentale, les années 1960 ont été marquées par une entrée massive des femmes mariées sur le marché du travail. Favorisée par une offre d'emplois accrue dans le secteur des services, et sans doute aussi par la récente mise en marché d'une contraception efficace, cette «sortie » des femmes et des mères hors du foyer va susciter, surtout en Amérique du nord, où les services de garde étaient quasi inexistants, des débats et des luttes qui s'incarneront, notamment, dans le mouvement des femmes ${ }^{\mathrm{I}}$.

À ce propos, on a souvent dit que le mouvement féministe, qui amorce sa seconde phase vers la

LIEN SOCIAL ET POLITIQUES - RIAC; 36 AUTOMNE 1996 même époque, a été porteur d'une idéologie qui valorisait le travail des femmes à l'extérieur de la maison, au détriment de leurs activités maternelles. Cette idée paraît assez répandue dans l'opinion publique et incite à poser la question: le mouvement féministe aurait-il « légitimé » un tel modèle, celui de la «mère-travailleuse »?

La réponse à cette question n'est pas univoque, le mouvement féministe ayant été, du moins à ses tout débuts, loin d'être unanime sur le sujet. Sans évidemment reprendre toute l'histoire des idées et des luttes du mouvement en cette matière depuis trente ans, les paragraphes qui suivent vont plutôt s'attarder à rappeler les premières analyses qui, au tournant des années 1970, c'est-à-dire cette courte période de la fin des années
1960 et du tout début des années 1970 , se sont révélées capitales dans l'articulation ultérieure de la pensée féministe sur ce sujet.

\section{Le féminisme libéral égalitaire :} oui au travail salarié

On s'entend pour dire que la seconde phase du féminisme ${ }^{2}$ fait son apparition au milieu des années 1960 aux États-Unis. C'est le livre de Betty Friedan, The Feminine Mystique, paru en 1963, qui lui conne son coup d'envoi. Ce n'est qu'à partir de ce moment que le monde des jeunes femmes découvre l'essai de Simone de Beauvoir, Le Deuxième Sexe, paru en 1949, en pleine période d'amnésie du féminisme. Betty Friedan dénonce la situation des femmes à la maison et propose comme solution à leur «malaise 
Le féminisme et la question des « mères travailleuses Retour sur le tournant des années 1970

qui n'a pas de nom » le travail à l'extérieur du foyer, y compris pour les mères, des garderies devant leur venir en aide à cet effet.

Pour Simone de Beauvoir, le travail à l'extérieur faisait partie du processus d'affranchissement des femmes, l'affranchissement total étant cependant subordonné à une révolution socialiste des moyens de production, ce qui était loin d'être le cas pour Betty Friedan. The Feminine Mystique est en effet le livre type du féminisme libéral égalitaire: c'est la socialisation et les préjugés qui expliquent le sort fait aux femmes, et non l'organisation économique. Ce sont les mentalités et les valeurs - rétrogrades dans le cas des femmes - qui forgent l'organisation sociale. Pour la féministe libérale Betty Friedan, l'organisation sociale est réformable par le biais de l'éducation et des lois. Plus les filles seront instruites, plus elles travailleront à l'extérieur et plus elles changeront le système: telle est la voie royale vers l'égalité. Des lois devront être modifiées pour faciliter l'insertion des femmes au marché du travail et des garderies devront être créées et mises à la disposition des mères qui voudront travailler à l'extérieur de la maison.

Ce féminisme libéral imprègne aussi les groupements de femmes qui se formeront à l'époque. Par exemple NOW (National Organization of Women) surgit aux États- unis en 1966, et la Fédération des femmes du Québec ici au même moment. Il imprègne aussi totalement l'important Rapport de la Commission royale d'enquête sur le statut de la femme au Canada, qui paraît en 1971.

Oui, ce féminisme-là faisait vraiment du travail salarié des femmes la voie vers l'«émancipation» et le tremplin vers l'égalité. Il s'agissait pour les femmes de s'assimiler au monde - masculin en l'accurrence - du travail.

\section{L'arrivée des groupes féministes radicaux}

Puis, aux États-Unis, sont apparues presque tout de suite après, c'est-à-dire en 1967-1968, des femmes provenant du mouvement étudiant, des «Civil Rights » et de la «New Left», des dissidentes de NOW, qui formèrent de petits groupes autonomes (d'où les hommes étaient exclus d'emblée) appelés Women's Liberation Groups, ou plus familièrement «Women's Lib». Ils prendront le nom de MLF (Mouvement de libération des femmes) en France en $1970^{3}$, de FLF (Front de libération des femmes) au Québec (1969) ${ }^{4}$. Leur cheminement commun fut bien décrit par Juliet Mitchell à l'époque dans Women's Estate (Mitchell, 1974).

Dans la foulée de la démocratisation de l'éducation, ces femmes s'étaient instruites et voyaient ce que valait, en réalité, l'égalité tant promise sur le marché du travail : le revenu des femmes ne représentait en 1967, tout au moins au Québec, que 51,2 pour cent de celui des hommes (Bisson, 1987: 9) ${ }^{5}$. Elles expérimentaient aussi ce que signifiait cette égalité dans la res publica à l'occasion de leur participation aux mouvements de contestation sociale. La place qu'on leur assignait invariablement était celle de subalternes: colleuses de timbres, fabricantes de pancartes, confec- tionneuses de goûters et serveuses de café. La pilule contraceptive, devenue accessible, était censée les libérer des maternités involontaires pour enfin les faire accéder à l' «amour libre», mais cela correspondait aussi, dans les faits, à une déresponsabilisation des hommes en matière de sexualité. Comme le dit Juliett Mitchell :

L'idéologie égalitariste ne sert pas à masquer l'écart entre l'illusion et la réalité, mais est justement le mode sur lequel la discrimination, autant que son refus, sont vécus. Le fait de croire à la possibilité et à la justesse d'une telle égalité a permis aux femmes de se sentir trompées et a été la condition de base de leur protestation initiale (Mitchell, 1974: 46).

En un mot, elles avaient tout le loisir de constater autour d'elles que l'égalité était un leurre pour les femmes (et pour les hommes pauvres, comme le croyaient en plus celles qui alliaient leur féminisme à une perspective de classes). Il fallait trouver pourquoi.

L'horizon idéologique qui s'offrait, en 1968, 1969 et 1970 , aux jeunes militantes qui ont formé les rangs des premiers groupes féministes était grosso modo le suivant: il y avait d'un côté ce courant du féminisme libéral égalitaire qui prônait l'émancipation des femmes et des mères par le travail salarié. De l'autre, il y avait aussi le courant socialiste, du moins en France et au Québec (car l'horizon idéologique des Américaines fut à cet égard différent ${ }^{6}$ ); les références théoriques en la matière se résumaient principalement au livre d'Engels, L'Origine de la famille, de la propriété privée et de l'État, et à différents écrits nationalistes tiers-mondistes, dont le Portrait du colonisé d'Albert Memmi et les Nègres blancs d'Amérique de Pierre Vallières.

Pour les socialistes aussi, l'émancipation des femmes passait par le travail salarié. Engels parlait de «l'affranchissement de la 


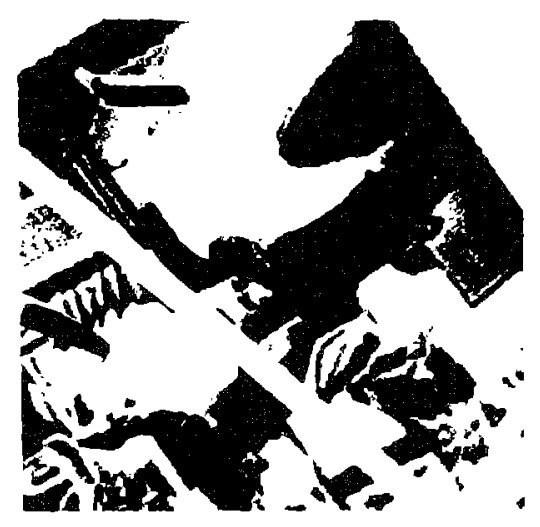

femme et de sa condition première : la rentrée de tout le sexe féminin dans l'industrie publique » comme solution à leurs problèmes. La seule lutte légitime des femmes passait donc par la lutte sur le front du travail salarié, côte à côte avec les camarades. Toute lutte «autonome» de fermmes était à cet égard contrerévolutionnaire et divisait la classe ouvrière: elle était proprement «illégitime».

\section{La remise en question du mythe de la libération par le travail salarié}

L'arrivée des nouveaux groupes féministes sur la scène politique remettra en question ce mythe de l'égalité et de la libération par le travail salarié. La féministe italienne Mariarosa Dalla Costa commente la question en ces termes: «L'avènement du mouvement des fermmes est le refus de cette alternative [...] Le mouvement ne peut se développer qu'en opposition à cette alternative. $\mathrm{Par}$ son existence même, il affirme et devra affirmer, par une action toujours plus cohérente, le refus, de la part des femmes, du mythe de la libération par le travail. Nous avons assez travaillé » (Dalla Costa, 1973: 95).

À peu près simultanément, entre 1968 et 1972, dans des pays occidentaux tels que les États-Unis, la France, 1'Italie, l'Angleterre, le Canada et le Québec, sont en effet publiées des analyses, produites par des militantes de ces groupes féministes, qui essaient de comprendre les raisons de la place secondaire occupée par les femmes à l'extérieur de la maison. Le regard est à cet effet tourné vers l'immense travail que les femmes effectuaient déjà à la maison dans la sphère familiale. On peut rappeler certains titres, publiés entre 1968 et 1972 , qui tous deviendront ultérieurement des «classiques» de la question: Mainardi (1970), Benston (1969), Larguia (1970), Morton (1970), Dupont (1970), Warrior (1973 [1971]), Dalla Costa (1973 $\left.[1971]^{7}\right)$.

Pour le Québec, on peut signaler la publication en 1972 d'une Analyse socio-économique de la ménagère québécoise. Il s'agit d'une première tentative de compilation globale de données sur la situation des ménagères à temps plein $^{8}$ au Québec à la lumière du recensement de 1971. L'ouvrage comprend aussi une sorte d' «état des lieux» de la recherche sur la question ${ }^{9}$.

Ces textes ne résument pas la totalité de la production féministe de cette période, loin de là. Il s'agit plutôt d'un échantillon de ceux qui sont à l'origine de l'émergence publique d'une figure jusqu'alors invisible: la femme mariée, son travail à l'intérieur de la famille et son exploitation. C'est en effet à partir de ces écrits que l'on considérera que les femmes vivent dans la famille une oppression "spécifique », qu'elles y effectuent un travail d'entretien matériel et «immatériel» (affectif). On tente de comprendre les raisons de la gratuité de ce travail. Et on découvre là un rapport social précis, un rapport social de sexe, et un rapport de production particulier (Dupont, 1970). On découvre aussi que les types de travaux que les femmes exécutent à l'extérieur de la maison sont en lien direct avec le travail qu'elles effectuent au foyer. On parle même d' «extension des travaux ménagers ». On se rendra compte à cet égard que, si le travail des femmes (comme infirmières, éducatrices, serveuses, cuisinières, bonnes, etc.) sur le marché du travail est si mal payé, c'est qu'elles effectuent souvent le même à la maison, mais gratuitement.

\section{Une légitimation de la révolte et de la lutte autonome des femmes}

Bref, en mettant ainsi en évidence le travail des femmes dans la famille, ces analyses se sont trouvées à changer les termes et les axes de l'analyse traditionnelle du travail et de la famille. La relecture de cette documentation du début des années 1970 a incité certaines analystes à parler à cet égard de «double rupture »:

l'une «épistémologique » (comme on disait à l'époque) qui, à partir de l'analyse du travail foumi par les femmes, a tenté de modifier les conceptions sociologiques traditionnelles du travail et de la société ; l'autre, politique, qui, déplaçant les lieux de l'exploitation des femmes jusque dans l'intimité des foyers et des alcôves affirmait avec vigueur que «le privé est politique», contestant ainsi radicalement (au moins pour quelque temps) les formes usuelles de l'analyse, de l'intervention et de l'action politiques (Chaudron et al., 1984: 181).

C'est en effet cette «découverte » de l'oppression «spécifique » cles femmes dans la famille même - donc en dehors de la sphère traclitionnelle «légitime» de lutte: le front du travail salarié - qui a légitimé, à l'époque, cette nouvelle forme d'intervention et d'action politiques: la lutte autonome des femmes.

Il paraît donc difficile d'affirmer que le féminisme du début des années $1970 \mathrm{a} \mathrm{pu}$, en bloc, «légitimer» le modèle de la mère-travailleuse salariée (même si toutes ces féministes, mères ou non, devaient travailler pour gagner 
Le féminisme et la question des $\alpha$ mères travailleuses Retour sur le tournant des années 1970
On peut donc conclure que si cette section du féminisme qui a dénoncé la double journée des femmes a légitimé quelque chose dans ce domaine, c'est plutôt la révolte des femmes contre cette «condition » (d'où le slogan «On a raison de se révolter »), puis la lutte pour changer cette condition et les rapports sociaux qui l'engendraient. C'est donc bien davantage cette révolte et cette lutte que le féminisme du tournant des années 1970 aurait légitimées, plutôt que le modèle de la mère-travailleuse et le mythe de la libération par le travail salarié. D'ailleurs, à la suite de ces analyses radicales de la place des femmes dans la société, la question du travail des femmes à l'intérieur et à l'extérieur de la famille ne se posera plus - tout au moins pour les courants marquants de la pensée féministe - dans les termes étroits de la problématique libérale de Betty Friedan ou de la pensée marxiste orthodoxe ${ }^{12}$.

\section{Aujourd'hui, des résultats décevants}

À partir des années 1970 mouvement aura justement à son programme de dégonfler le mythe de la libération qui y était attaché, voire, pour un certain courant international, d'aller jusqu'à revendiquer, à partir de 1972-1973, la salarisation du travail ménager, qui avait déjà été revendiquée par des féministes du début du siècle ${ }^{10}$.

Parti d'Italie en 1971, ce courant suscita en effet la formation de collectifs en Europe (Angleterre, Allemagne, Suisse), aux États-Unis et au Canada anglais, collectifs qui se constituèrent en un Réseau international du salaire au travail ménager. Si ses analyses connurent durant la décennie 1970 une grande popularité, la stratégie qui en découlait, soit un salaire pour le travail ménager (et non un salaire «à la ménagère»), a cependant été rejetée par l'ensemble du mouyement ${ }^{11}$. total. L'effet «boomerang » de ces piètres résultats retombe d'ailleurs sur tout le mouvement féministe, qui se voit maintenant, ici et là, blâmé pour son incapacité à faire bouger et à changer l'ordre sociosexuel dominant.

Pour sa part, l'Organisation internationale $\mathrm{du}$ travail posait, encore récemment, un constat implacable: «Dans la quasi-totalité des pays du monde, les femmes travaillent plus dur tout en étant moins bien payées que les hommes. Et l'écart se creuse même dans certains pays ${ }^{13} \gg$. La question de la reconnaissance du travail de reproduction sociale assumé par les femmes à l'intérieur et à l'extérieur de la famille, et sur toute la surface de la terre, demeure donc entière. On qualifie ce travail, dans certaines études, de véritable «subvention, occulte et occultée, qui permet à l'économie nationale de fonctionner » (Neptune Anglade, 1986: 15$)^{14}$.

À mesure que des études empiriques sur le thème de la «conciliation travail salarié-travail domestique» sont publiées en Occident ${ }^{15}$, on constate que la question du travail de reproduction peut de moins en moins être résolue privément au moyen de certaines stratégies mises de l'avant par les femmes salariées, comme le partage des tâches et le recours au personnel domestique féminin. On redécouvre, maintenant, que la question du travail domestique, «ce n'est pas seulement du temps gratuit à répartir autrement, c'est une organisation sociale définissant la place des femmes » (FougeyrollasSchwebel, 1994-1995 : 41).

Comme le remarquait à juste titre récemment Jan Windebank en conclusion de son analyse des débats français autour du travail domestique des 25 dernières années, la recherche empirique en la matière ne doit pas ignorer la nature éminemment politique de 
cette question: «On ne doit pas perdre de vue la motivation initiale de son étude, à savoir expliquer le rapport des femmes au foyer et à la famille» (Windebank, 1994 : 32).

\section{De la remise en question des rapports de sexe à la «gestion de la double tâche »}

Bref, il ne faudrait pas perdre de vue, dans ces recherches empiriques, la question première qui fut à l'origine de tout ce questionnement féministe contemporain: pourquoi les femmes ont-elle la charge de la maisonnée et de la famille? «Décider pourquoi s'instaure cette situation», dit encore J. Windebank, «a bien évidemment des implications importantes pour trouver des voies qui permettraient aux femmes de changer cette situation ». En effet, un tel questionnement permet de situer ces voies de changement dans une perspective de remise en question des rapports de sexe et de l'assignation des femmes à la famille, et non plus seulement dans une perspective de «gestion de la double tâche», optique qui, de plus en plus, prévaut en la matière de part et d'autre de l'Atlantique.

C'est d'ailleurs ce qu'a pu constater Annie Junter-Loiseau en analysant l'évolution des différents usages de la notion de «conciliation entre vie professionnelle et vie familiale $»$ dans les divers documents d'orientation consacrés à la politique sociale de l'Union européenne. Selon elle, l'utilisation de cette notion est la «réactualisation d'une vieille notion dont le sens a glissé de la "conciliation des rôles féminins" vers "la conciliation de la vie professionnelle et familiale" $\gg$. Elle participerait même de la «réorientation de la politique d'égalité entre les femmes et les hommes du champ du droit social où elle tentait de s'enraciner, vers celui de la gestion des ressources

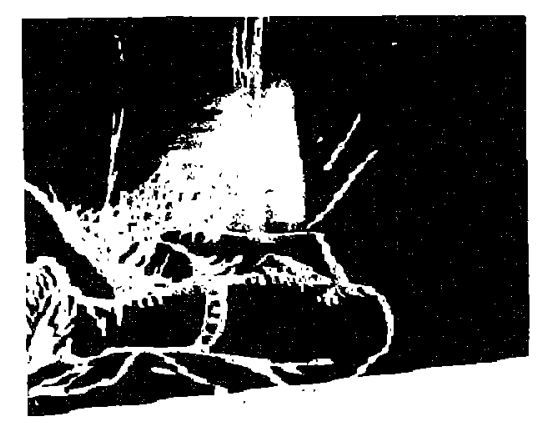

plus radicaux du féminisme qui étaient porteurs d'une remise en question du modèle libéral de la libération des femmes par le travail salarié.

Louise Toupin

Institut Simone de Beauvoir Université Concordia

humaines ». Pour cette juriste, «la question de la redistribution des rôles familiaux n'est pas réductible à un mode de gestion " (Junter-Loiseau, 1995: 11).

À la lumière du large débat sur le travail de reproduction déclenché par les féministes au tournant des années 1970 , on peut certes regretter, dans certaines orientations actuelles, ce qu'il faut bien appeler un "rétrécissement de la problématique». On doit surtout déplorer que cette optique de «gestion de la double tâche» soit en passe de devenir progressivement l'unique perspective de réforme préconisée à l'endroit des femmes salariées en situation conjugale.

La mise en parallèle de ces récentes orientations avec les orientations de départ ne remet pas en question la valeur et la pertinence de ce type de recherches; elle permet toutefois de les situer historiquement, et donc de mettre en relief le fait que d'autres perspectives ont existé et, enfin, que d'autres doivent demeurer ouvertes à la recherche ${ }^{16}$.

Pour finir, on peut se demander, rétrospectivement, si la mise à l'écart progressive des orientations définies par ces groupes du tournant des années 1970 n'a pas eu un «effet pervers»: celui de contribuer à faire oublier peu à peu l'existence même de ces courants

Selon Mariarosa Dalla Costa, deux conditions ont permis cette entrée massive des femmes sur le marché du travail. Elles ont, en premier lieu, "renonc[é] à avoir des enfants, et par là, refus[é] certaines tâches et responsabilités familiales »et, en deuxième lieu, « appel[é] à titre gratuit, ou sous-payé, d'autres femmes à effectuer les travaux domestiques. C'est-à-dire que le travail féminin extérieur ne devenait possible qu'au prix de la réduction du travail domestique à ses moindres termes, ou en établissant une stratification ultérieure parmi les femmes. La contradiction du destin féminin débouchait sur la fondation d'une autre contradiction, et done restait sans solution. $\grave{A}$ ce vieux problème, non seulement ne répondait aucune gammi de stratégies mais, par rapport au passé, les quelques stratégies tentées étaient loin d'être novatrices " (Dalla Costa, 1988 : 123).

2 La première phase, échelonnée grosso modo sur un siècle, se terminerait avec le début des années 1960.

3 Sur l'appropriation du sigle MLF par Antoinette Fouque et son groupe, voir Chronique d'une imposture (1981), et Picq, 1993: 292-311.

4 Sur les tout débuts du néo-féminisme québécois, voir O'Leary et Toupin, 1982.

5 Il s'agit ici du revenu de I'ensemble des hommes et des femmes travaillant à temps plein ou à temps partiel, en 1967. Lorsqu'on établit la distinction «temps plein » et «temps partiel », le revenu moyen d'emploi à temps plein des femmes s'établit à environ 56 pour cent

6 Comme le dit Juliett Mitchell : «Le MLF aux États Unis avait ceci de particulier par rapport aux autres mouvements qui se développèrent plus tard dans les autres pays qu'il ne subissait aucune influence du mouvement ouvrier et qu'il n'était pas d'accord avec le postulat marxiste de la classe ouvrière comme seule classe révolutionnaire. Les femmes marxistes du Mouvement étaient surtout des étudiantes, dans un pays où la ligne de partage social sem- 
blait passer davantage par la race que par la classe » (Mitchell, 1974: 61-62).

C'est d'ailleurs parmi les femmes noires que l'on retrouvera un fort contingent de féministes partageant une perspective de classes et tentant d'allier, dans leurs analyses, les dimensions sexe, "race » et classe. C'est surtout après 1973, date de la formation du premier regroupement féministe noir «séparé ", le National Black Feminist Organisation (NBFO), que s'implantera le «Black Feminism» comme courant autonome et que s'élaborera ce qui sera appelé plus tard le «Black Feminist Thought $»$. Voir, entre autres, Combahee River Collective, 1983 ; Davis, 1983 ; Hooks, 1981 ; et Hill Collins, 1990.

7 Écrit en 1971, publié en Italie et aux ÉtatsUnis au début de 1972, puis à Londres à la fin de 1972 (sous le titre Women Power and the Subversion of the Community, accompagné d'un texte de Selma James, «La place des femmes », écrit en 1953), le texte a paru en français et en allemand en 1973. Il a ensuite été traduit en espagnol en 1975 et en japonais en 1986. Notons qu'il fut écrit sans connaissance préalable des autres textes sus-mentionnés, ce qui laisse supposer que « le temps était mûr, en Italie et à l'étranger, pour l'explosion de cette question » (entrevue de Mariarosa Dalla Costa avec l'auteure de ces lignes, mars 1996).

8 Le terme «ménagère » peut paraître à certains aujourd'hui suranné. Il est utilisé ici pour respecter l'esprit et la terminologie de l'époque, et aussi pour éviter certaines confusions inherentes à d'autres appellations. Comme I'a signalé Louise Vandelac dans le Document synthèse d'une vaste recherche sur la production domestique : « L'emploi du terme travailleuse au foyer risque en effet de semer la confusion entre les ménagères et les travailleuses à domicile qui effectuent un travail résnunéré, déclaré ou non, à la maison. Le terme travailleuse domestique risque, de son côté, d'être confondu avec celui d'employée de maison (bonne, femme de ménage, gardienne, etc.) ( Vandelac, 1983: 136). En France, on semble avoir préféré le terme « femme au foyer ». Au Québec, l'Association féminine d'éducation et d'action sociale l'AFEAS, le principal regroupement de femmes qui lutte pour faire inclure ce travail dans le PNB, a pour sa part opté, ces dernières années, pour le terme «travailleuse au foyer ».

9 Cette analyse est le fruit d'un collectif de femmes formé en grande partie de militantes du Front de libération des femmes au moment de la dissolution de ce groupe à l'automne 1971. Voir Benoit et al,, 1972.

${ }^{10}$ Voir entre autres à ce sujet Schirmacher, 1904 ; Bock et Duden, 1980 ; Roussel, 1978 ; Rathbone, 1986 ; Bock, 1992 ; Cova, 1994.

${ }^{11}$ Ce qui n'a pas empêché, et n'empêche toujours pas, nombre de femmes à la maison de considérer qu'elles effectuent là un véritable travail qui mérite reconnaissance, sinon rémunération. C'est du moins ce discours que tenaient, et que tiennent toujours. des femmes peu scolarisées, bénéficiaires d'allocations d'aide sociale (Dandurand, 1986 ; Bélanger, 1990). Car l'emploi salarié qu'elles peuvent occuper sur le marché du travail, n'étant ni très qualifié, ni bien rémunéré, ni très satisfaisant, peut difficilement et rarement, sauf exception évidemment, être assimilé à une quelconque « libération », C'est d'ailleurs ce que certaines voix de fernmes noires avaient fait entendre au tout début du renouveau féministe aux États-Unis. Ainsi, selon Margaret Wright : «Les Noires ont été doublement opprimées. Dans le travail, nous sommes à l'échelon le plus bas. Les Blanches ont leurs problèmes. On leur offre des postes de secrétaires et non de direction. Mais nous, on nous propose de laver les parquets et autres besognes. Quelquefois, on nous donne les restes du frigo. On est tout le temps exploitées. Et quand on rentre chez soi, le vieux se demande pourquoi la soupe n'est pas prête " (cité dans Lerner, 1975 : 347).

L'absence de sensibilisation de nombre de féministes américaines blanches aux problèmes vécus par les femmes pauvres, sous-scolarisées et démunies, et particulièrement par les femmes « de couleur», a constitué un facteur clé de la réticence de ces demières à se joindre au mouvement de libération des femmes à ses tout débuts. soit durant la période étudiée ici. Voir Hook, 1981 : 188.

Sur le courant du salaire au travail ménager, à part Dalla Costa et James (1973; voir bibliographie, sous Dalla Costa), on peut consulter, en français, Collectif l'Insoumise (1977). J'effectue en ce moment une recherche sur l'histoire du Réseau international du salaire au travail ménager.

12 Pour une analyse des étapes qui ont jalonné l'évolution du système de pensée féministe d'inspiration matérialiste, voir la première partie de Juteau et Laurin, 1988, et Battagliola et al., 1990.

13 Voir La Fresse, 8 septembre $1992:$ A14.

${ }^{14}$ La demière conférence mondiale de l'ONU sur les femmes, tenue à Beijing en septembre 1996, a d'ailleurs inclus, dans son programme d'action, un paragraphe (165g) incitant les divers gouvernements à prendre en considération ce travail de reproduction dans leur comptabilité nationale.

${ }^{15}$ Les notions utilisées récemment pour analyser cette question, qualifiée au début des années 1970 de « double exploitation " des femmes, yarient sensiblement selon les pays : "articulation travail-maternité » ou «conciliation vie professionnelle et familiale » en France et au Québec (Dandurand et Descarries, 1992 ; Méthot et Vandelac, 1993), « vie en double » (BarrèreMaurisson, 1992), "doppia presenza" (double présence) en Italie (Bimbi, 1989).

${ }^{16}$ Les perspectives développées par l'équipe du GEDISST (Groupe d'études sur la division sociale et sexuelle du travail), un laboratoire du CNRS à Paris, à partir de la notion de «rapports sociaux de sexe » sont ici à signaler. Une introduction à cette perspective se retrouve dans Battagliola (1990).

\section{Bibliographie}

BARRERE-MAURISSON, Marie-Agnès 1992. La Division familiale du travail. La vie en double. Paris, PUF, $256 \mathrm{p}$.

BATTAGLIOLA, Françoise, et al. 1990 [1986]. À propos des rapports sociaux de sexe : parcours épistémologiques. Paris, Centre de sociologie urbaine, 252 p.

BÉLANGER, Lucie. 1990. «Alternative à l'appauvrissement des femmes ", UniversElles, 3,1 (novembre) : 4-5.

BENOIT, Carmelle, et al. 1972. Analyse socio-économique de la ménagère québécoise. Montréal, Centre de recherche sur la femme, $287 \mathrm{p}$.

BENSTON, Margaret. 1969. «The Political Economy of Women Liberation ». En traduction française (1970) : «Pour une économie politique de la libération des femmes », Partisans, 54-55, juilletoctobre : $23-31$.

BIMBI, Franca. 1989. " "The Double Presence" : A Complex Model of Italian Women's Labor ", Marriage and Family Review, 14, 1-2: 81-105.

BISSON, Louise. 1987. Le Salaire a-t-il un sexe? Les inégalités de revenus entre les femmes et les hommes au Québec. Québec, Les Publications du Québec, $90 \mathrm{p}$. 
BOCK, Gisela. 1992. «Pauyreté féminine, droits des mères et États-providence », dans Françoise THEBAUD, dir. Histoire des femmes en Occident, 5 : Le XXe siècle. Paris, Plon, chapitre 13: 381-409.

BOCK, Gisela, et Barbara DUDEN. 1980. «Labor of Love/Love as Labor: On the Genesis of Housework in Capitalism ", dans From Feminism to Liberation. Cambridge, Mass., Shenkman Publishing Co., édition révisée.

CHAUDRON, Martine, et al. 1984. "Féminisme et analyses du travail des femmes », dans Les Femmes et la question du travail. Centre lyonnais d'études féministes. Lyon, Presses universitaires de Lyon: 175-192.

Chronique d'une imposture: du Mouvement de libération des femmes à la marque commerciale. Paris, Tierce, 1981

COLLECTIF L'INSOUMISE. 1977. Le Foyer de l'insurrection. Textes sur le salaire pour le travail ménager. Genève.

COMBAHEE RIVER COLLECTIVE. 1983 "The Combahee River Collective Statement », dans Barbara SMITH, éd. Home Girls. A Black Feminist Anthology. New York, Kitchen Table : 272-282.

COVA, Anne. 1994. Droits des femmes et protection de la maternité en France, 1892 1939. Florence, Institut universitaire européen, Département d'histoire et civilisation, thèse de doctorat,

DALLA COSTA, Mariarosa. 1973 [1971] «Donne e sovversione sociale " (1971) version française dans Mariarosa DALLA COSTA et Selma JAMES. Le Pouvoir des femmes et la subversion sociale. Genève. Librairie Adversaire : 41-97.

DALLA COSTA, Mariarosa, 1988. «La femme entre la famille et les politiques d'emploi en Italie ", Les Cahiers de l'APRE , 7, avril-mai : 121-127.

DANDURAND, Renée B. 1986. «Identité sociale et maternité sans alliance », dans Identités féminines: mémoire et création. Québec, Institut québécois de recherche sur la culture, Questions de culture, 9: 85103.

DANDURAND, Renée B., et Francine DESCARRIES, dir. 1992. Mères et travailleuses. De l'exception à la règle. Québec, Institut québécois de recherche sur la culture, $214 \mathrm{p}$.

DAVIS, Angela 1983. Femmes, race et classe. Paris, Des femmes.

DUPONT (DELPHY), Christine. 1970. «L'ennemi principal », Partisans, 54-55, juillet-octobre : $157-172$.

FOUGEYROLLAS-SCHWEBEL, Dominique. 1994-1995. « Si la famille m'était comptée $»$, Politis, la revue, 8 (novembredécembre-janvier) : 37-41.
HILL COLLINS, Patricia. 1990. Black Feminist Thought: Knowledge, Consciousness, and the Politics of Empowerment. New York, Routledge.

HOOK, Bell. 1981. Ain't I A Woman? Black Women and Feminism. Boston, South End Press, 205 p.

JUNTER-LOISEAU, Annie. 1995. La Notion de conciliation dans les travaux $d u$ Forum. Communication présentée à la conférence de clôture du Forum européen «Gender et emploi du temps », 23-24 juin, Florence, Institut universitaire européen, $15 \mathrm{p}$.

JUTEAU, Danielle, et Nicole LAURIN. 1988. «L'évolution des formes de l'appropriation des femmes: des religieuses au "mères porteuses" ", Revue canadienne de sociologie et d'anthropologie/Canadian Review of Sociology and Anthropology, $25,2: 183-192$

LARGUIA, Isabel. 1970 [1969]. « Contre le travai] invisible *, Partisans, 54-55, juillet-octobre : 206-220 (d'abord paru en anglais).

LERNER, Gerda. 1975. De l'esclavage à la ségrégation. Les femmes noires dans l'Amérique des Blancs. Paris, Denoël/ Gonthier, $350 \mathrm{p}$

MAINARDI, Pat. 1970 [1968]. "The Politics of Housework », dans Robin MORGAN, éd. Sisterhood is Powerfull. New York, Vintage Books : 447-443.

MÉTHOT, Andrée-Lise, et Louise VANDELAC dir. 1993. Concilier... l'inconciliable. La conciliation des responsabilités familiales et professionnelles dans trois milieux de travail de la région de Montréal. Montréal, Université du Québec à Montréal, Centre pour l'étude des interactions biologiques entre la santé et l'environnement, mars, $61 \mathrm{p}$.

MITCHELL, Juliet. 1974. L'Âge de femme. Paris, Des femmes, $233 \mathrm{p}$.

MORTON, Peggy. 1970. «A Woman's Work Is Never Done », Leviathan, II, 1, 1970 : 32-37. Version revue et augmentée dans Edith HOSHINO ALTBACH, éd. 1980. From Feminism to Liberation. Cambridge, Mass., Schenkman Publishing : 243-260.

NEPTUNE ANGLADE, Mireille. 1986 $L$ 'Autre Moitié du développement. $\dot{A}$ propos du travail des femmes en Haiti. Montréal et Port-au-Prince, Éd. des Alizés et ERCE. $261 \mathrm{p}$.

O'LEARY, Véronique, et Louise TOUPIN, dir. 1982. Québécoises Deboutte! Une anthologie de textes du Front de libération des fermmes (1969-1971) et du Centre des femmes (1972-1975). Montréal, Éd. du Remue-ménage.

PICQ, Françoise. 1993. Libération des femmes: les années-mouvement. Paris, Seuil : 292-311.
RATHBONE, Eleanore. 1986 [1924]. The Desinherited Family. Bristol, Falling Wall Press, $400 \mathrm{p}$.

ROUSSEL, Nelly. 1979 [1906]. L'Éternelle Sacrifiée. Paris, Syros, 112 p.

SCHIRMACHER, Kaëthe. 1904. « Le travail domestique des femmes. Son évaluation économique et sociale ", Revue d'économie politique, $18: 353-379$.

VANDELAC, Louise. 1983. Document-synthèse. Document 1. Production domestique. Gouvernement du Québec, Conseil du statut de la femme, $220 \mathrm{p}$

VANDELAC, Lauise, dir. 1985. Du travail et de l'amour. Les dessous de la production domestique. Montréal, Saint-Martin, $418 \mathrm{p}$.

WARRIOR, Betsy. 1973 [1971]. « Housework : Slavery or Labour of Love », dans Anne KOEDT et al. Radical Feminism. New York, Quadrangle : 208212.

WINDEBANK, Jan. 1994. «Comment expliquer le rapport des femmes au foyer et à la famille : les débats français autour du travail domestique ", Nouvelles Questions féministes, 15, 1:9-34. 